\title{
Spatio-temporal dynamics induced by competing instabilities in two asymmetrically coupled nonlinear evolution equations
}

\author{
D. Schüler, ${ }^{1}$ S. Alonso, ${ }^{1}$ A. Torcini, ${ }^{2,3}$ and M. Bär ${ }^{1}$ \\ ${ }^{1}$ Physikalisch-Technische Bundesanstalt, Abbestrasse 2-12, 10587 Berlin, Germany \\ ${ }^{2}$ CNR-Consiglio Nazionale delle Ricerche, Istituto dei Sistemi Complessi - Via Madonna del Piano 10, \\ I-50019 Sesto Fiorentino, Italy \\ ${ }^{3}$ INFN Sez. Firenze, via Sansone 1, I-50019 Sesto Fiorentino, Italy
}

(Received 22 August 2014; accepted 11 December 2014; published online 31 December 2014)

\begin{abstract}
Pattern formation often occurs in spatially extended physical, biological, and chemical systems due to an instability of the homogeneous steady state. The type of the instability usually prescribes the resulting spatio-temporal patterns and their characteristic length scales. However, patterns resulting from the simultaneous occurrence of instabilities cannot be expected to be simple superposition of the patterns associated with the considered instabilities. To address this issue, we design two simple models composed by two asymmetrically coupled equations of non-conserved (Swift-Hohenberg equations) or conserved (Cahn-Hilliard equations) order parameters with different characteristic wave lengths. The patterns arising in these systems range from coexisting static patterns of different wavelengths to traveling waves. A linear stability analysis allows to derive a two parameter phase diagram for the studied models, in particular, revealing for the Swift-Hohenberg equations, a co-dimension two bifurcation point of Turing and wave instability and a region of coexistence of stationary and traveling patterns. The nonlinear dynamics of the coupled evolution equations is investigated by performing accurate numerical simulations. These reveal more complex patterns, ranging from traveling waves with embedded Turing patterns domains to spatio-temporal chaos, and a wide hysteretic region, where waves or Turing patterns coexist. For the coupled CahnHilliard equations the presence of a weak coupling is sufficient to arrest the coarsening process and to lead to the emergence of purely periodic patterns. The final states are characterized by domains with a characteristic length, which diverges logarithmically with the coupling amplitude. (C) 2014
\end{abstract} AIP Publishing LLC. [http://dx.doi.org/10.1063/1.4905017]

Some chemical and biological systems exhibit competing pattern forming instabilities with different characteristic wave numbers. Often such a phenomenon is caused by the presence of different physical processes that appear on different length scales and cause patterns with different wavelengths. Here, we investigate two coupled SwiftHohenberg (SH) equations as well as two coupled CahnHilliard (CH) equations as minimal models for such multiscale pattern formation. The $\mathrm{CH}$ and the $\mathrm{SH}$ equations are partial differential equations describing the evolution of a conserved and a non-conserved order parameter, respectively. While the spatial domains in the $\mathrm{SH}$ equation self-organize into stationary periodic structures, for the $\mathrm{CH}$ equation the domains exhibit a coarsening dynamics that finally yield a single large domain. The competition between two instabilities with different wavelengths $\lambda_{1}$ and $\lambda_{2}$ is analyzed for coupled SH equations as well as for coupled $\mathrm{CH}$ equations. In both cases, the coupling of equations with stationary instabilities (Turing or phase separation) can lead to wave dynamics. Moreover, coupled SH equations exhibit a region of coexistence of Turing and traveling patterns as well as more complex patterns. The coupling of two $\mathrm{CH}$ equations leads to the arrest of coarsening and to the emergence of spatially periodic patterns.

\section{INTRODUCTION}

Reaction-diffusion equations are often employed to model systems outside of thermodynamic equilibrium. In some cases, these systems self-organize to form spatiotemporal structures. ${ }^{1}$ Prominent examples of such phenomena are oscillatory chemical reactions: e.g., the BelousovZhabotinsky (BZ) reaction produces oscillations and waves in the concentration of the involved chemical species and the chlorine dioxide-iodine-malonic acid (CDIMA) reaction produces stationary (Turing) patterns. ${ }^{2}$ Similar concepts of selforganization have been applied to explain different phenomena in biology. 3,4

The linear stability analysis of such equations may reveal possible instabilities in reaction-diffusion systems. Simple chemical and biochemical reactions can become unstable via a Hopf bifurcation and produce oscillatory behaviour. The addition of a spatial coordinate allows for the diffusion of the species and may produce stationary periodic patterns via a Turing instability ${ }^{5}$ or the emergence of (traveling) waves via a wave instability. ${ }^{5,6}$ Codimension-two bifurcations correspond to particular combinations of the parameter values where two types of instability appear simultaneously. In the proximity of such points, the associated dynamics have been extensively studied in the case of Turing-Hopf $^{7-10}$ and Turing-Turing ${ }^{11,12}$ codimension-two bifurcation and analyzed for the Turing-wave codimension- 
two bifurcation in a few instances. ${ }^{13,14}$ In particular, in all these cases, two instabilities appear simultaneously with two characteristic spatial scales, which can be very different depending on the parameter values. Such bifurcations appear in the BZ reaction, ${ }^{13}$ in catalytic surfaces with promotors, ${ }^{15}$ in the modelization of vegetation patterns in drylands, ${ }^{12}$ as well as in models of lipid domain formation in biomembranes. ${ }^{16,17}$ The interaction between different types of phospholipids and proteins on the membrane of living cells induces a spatial instability of the homogeneous state with a short characteristic spatial scale. ${ }^{16}$ On the other hand, the translocation of membrane proteins to the cytosol, where they rapidly diffuse to a different location on the membrane, may cause a spatial self-organization of the proteins on a larger spatial scale. ${ }^{16,18}$ The coupling between these processes induces the emergence of oscillatory patterns at the membrane at the larger spatial scale. ${ }^{17}$ This novel aspect is a central motivation in setting up the models investigated in this paper.

The $\mathrm{SH}$ equation is a generic equation for a nonconserved order parameter, which originally was developed for describing the instability of Rayleigh-Benard convection. ${ }^{19,20}$ The SH equation was also applied to biology, for example, as a model of nonlocal coupling in biological systems describing neural tissues. ${ }^{21} \mathrm{~A}$ complex variant of the SH equation has been previously employed to describe the dynamics of class B lasers. ${ }^{22}$ The standard SH equation undergoes a Turing instability following the increase of a bifurcation parameter, examples of the emerging patterns are reported in Figs. 1(a) and 1(c) in two and one spatial dimensions. The structure of the SH equation permits a straightforward control of the spatial scale of the instability by adjusting the parameters entering in the equation.

The $\mathrm{CH}$ equation describes the process of phase separation in a system with mass conservation. ${ }^{23}$ The system spontaneously segregates into spatial domains which grow and coarse continuously, see Figs. 1(b) and 1(d) for 2D and 1D examples. The difference between the evolution of the single $\mathrm{SH}$ and $\mathrm{CH}$ equations with similar characteristics can be appreciated in Fig. 1.

Here, we employ two coupled $\mathrm{SH}(\mathrm{CH})$ equations to introduce two different spatial scales in a system with two non-conserved (conserved) order parameters. The two equations are connected by an asymmetric coupling which induces a repertoire of spatio-temporal evolutions. While a model with a symmetric coupling between the two equations could be derived from an energy functional using a variational approach, asymmetrically coupled systems represent an effective description. However, such asymmetric coupling permits us to generate simple and generic models where two spatial bifurcations compete and give rise to waves. The combination of several $\mathrm{CH}$ may describe the process of phase separation with three or more components. In particular, it could be of interest for the processes of lipid separation at membranes ${ }^{24}$ or of phase separation in block copolymers melt. ${ }^{25}$ The combination of two SH may mimic the coupling of two layers where Turing patterns appear. ${ }^{26,27}$

The present article is organized as follows. In Sec. II the studied models are introduced and the corresponding linear
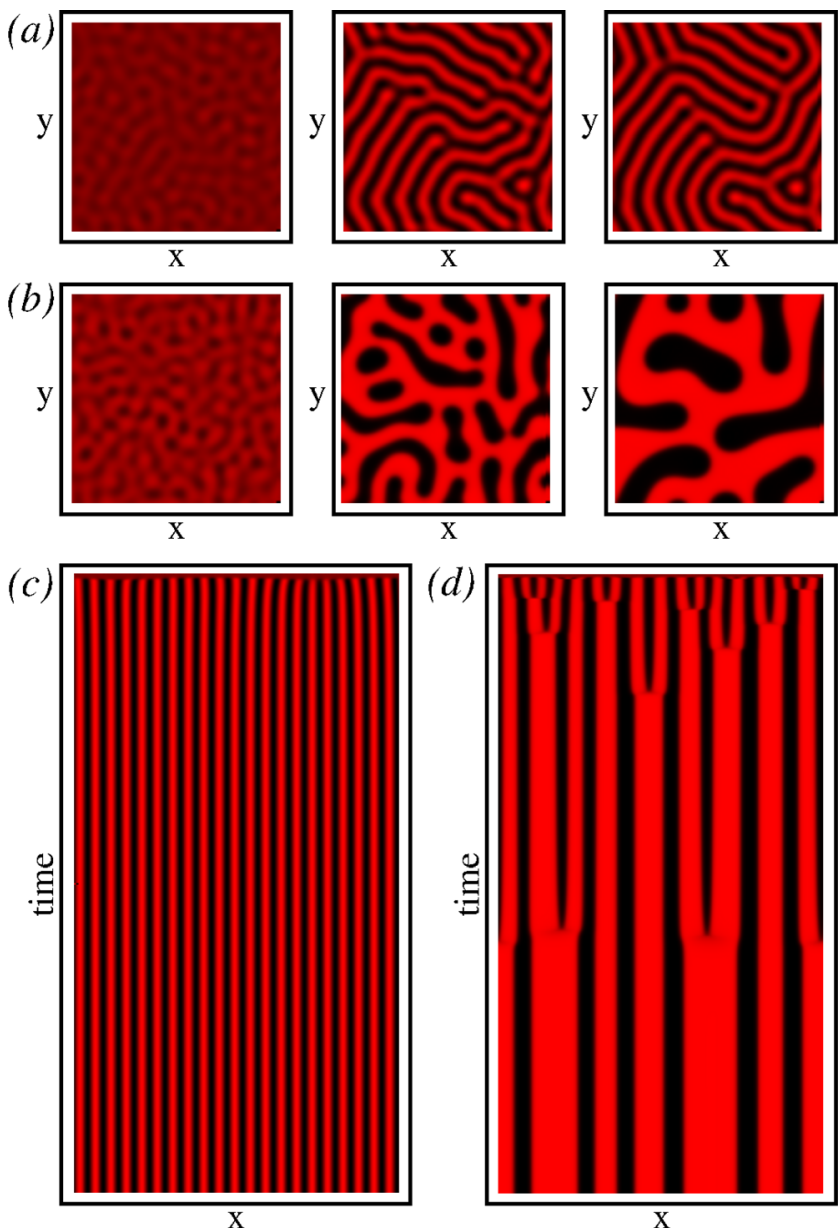

FIG. 1. Snapshots of a two-dimensional numerical simulations of a single SH equation (a) and a single $\mathrm{CH}$ equation (b) corresponding to times 8,40 , and 80 . The total size of the system is $31 \times 31$. Spatio-temporal plots of a onedimensional numerical simulations of a single SH equation (c) and a single $\mathrm{CH}$ equation (d), total time 3000 and total size $L=125$. Parameter values are $k_{1}=2$ and $\varepsilon=0.4$. Time direction is going downwards in (c) and (d).

stability analysis reported. Section III is devoted to the presentation of the results obtained in this study. In particular, the linear stability diagrams for the two models are described in Subsection III A, while Subsection IIIC reports detailed numerical investigations of the two models. Finally, the main results of this study are summarized in Sec. IV.

\section{MODELS}

Two simple models of multiscale pattern formation, based on the asymmetric coupling of two $\mathrm{SH}$ (resp. $\mathrm{CH}$ ) equations, are the main subjects of this article and are introduced in this section.

\section{A. Coupled SH equations}

We start considering the single SH equation, which was originally derived from the equations for thermal convection, but is commonly used as a generic model of pattern formation. $^{20,28}$ The SH equation exhibits static Turing patterns similar to the one observed for reaction-diffusion equations of activator-inhibitor type. In particular, the $\mathrm{SH}$ equation describes the spatial evolution of a single non-conserved dynamic variable $u$ 


$$
\frac{\partial u}{\partial t}=\varepsilon u-\left(\frac{1}{k_{1}^{2}} \nabla^{2}+1\right)^{2} u-u^{3},
$$

where the parameter $\varepsilon$ controls the linear stability of the homogeneous stationary solution (HSS) $u_{0}=0$ and the parameter $k_{1}$ is the critical characteristic wavenumber at the onset of instability at $\varepsilon=0$. One can easily determine the stability of the solution $u_{0}$ by the introduction of an infinitesimal spatially periodic perturbation, namely by considering $u=u_{0}+\delta u e^{\omega t-i k x}$. The resulting dispersion relation $\omega(k)$ is real-valued and it depends on the wavenumber $k$ as follows:

$$
\omega(k)=\varepsilon-1+2 \frac{k^{2}}{k_{1}^{2}}-\frac{k^{4}}{k_{1}^{4}} .
$$

When $\varepsilon>0$, the HSS become unstable for a finite interval of wavenumbers around $k_{1}$, namely, for $\sqrt{1-\sqrt{\varepsilon}} \leq k / k_{1}$ $\leq \sqrt{1+\sqrt{\varepsilon}}$. The expected characteristic spatial scale of the resulting Turing pattern is given by $\lambda_{1}=2 \pi / k_{1}$.

Next, we consider two linearly coupled SH equations, as simple model for multiscale pattern formation

$$
\begin{aligned}
& \frac{\partial u}{\partial t}=\varepsilon u-\left(\frac{1}{k_{1}^{2}} \nabla^{2}+1\right)^{2} u-u^{3}-\alpha v, \\
& \frac{\partial v}{\partial t}=\varepsilon v-\left(\frac{1}{k_{2}^{2}} \nabla^{2}+1\right)^{2} v-v^{3}+\alpha u .
\end{aligned}
$$

Each of these equations has a different characteristic length $\lambda_{i}=2 \pi / k_{i}$, with $i=1,2$. Furthermore, the same control parameter $\varepsilon$ for the instability is used in both equations ensuring that the instability occurs simultaneously in the decoupled systems. The coupling parameter $\alpha$ is the same in both equations. Note, however the opposite signs, which renders Eq. (3) non-variational, i.e., the dynamics of $u$ and $v$ in Eq. (3) cannot be derived as variational derivates of some functional $\mathcal{F}(u, v)$. We analyze the stability of the HSS $u_{0}=v_{0}=0$ by considering the following perturbations $u$ $=u_{0}+\delta u e^{\omega t-i k x}$ and $v=v_{0}+\delta v e^{\omega t-i k x}$. The linear stability analysis leads to the following dispersion relation:

$$
\begin{aligned}
\omega(k)= & \varepsilon-1+k^{2} \frac{k_{1}^{2}+k_{2}^{2}}{k_{1}^{2} k_{2}^{2}}-k^{4} \frac{k_{1}^{4}+k_{2}^{4}}{2 k_{1}^{4} k_{2}^{4}} \\
& \pm \sqrt{\left[k^{2} \frac{k_{2}^{2}-k_{1}^{2}}{k_{1}^{2} k_{2}^{2}}-k^{4} \frac{k_{2}^{4}-k_{1}^{4}}{2 k_{1}^{4} k_{2}^{4}}\right]^{2}-\alpha^{2},}
\end{aligned}
$$

which can produce spatial and spatio-temporal instabilities depending on the parameter values. For $\alpha=0$, the two equations become uncoupled and two real dispersion relations with maxima in $k_{1}$ and $k_{2}$ are obtained (see top panel of Fig. 2(a)). By coupling the two systems, the dispersion relation is modified. Nevertheless, the real part of the leading eigenvalue still resembles the respective curve for the uncoupled system with $\alpha=0$ and exhibits two maxima. The wavenumbers associated to these maxima will be the dominant modes of the coupled dynamics. We will indicate them as modes 1 and 2, corresponding to small and large wavenumbers, respectively. Furthermore, for sufficiently large coupling, the (a) Coupled SH equations

(b) Coupled $\mathrm{CH}$ equations
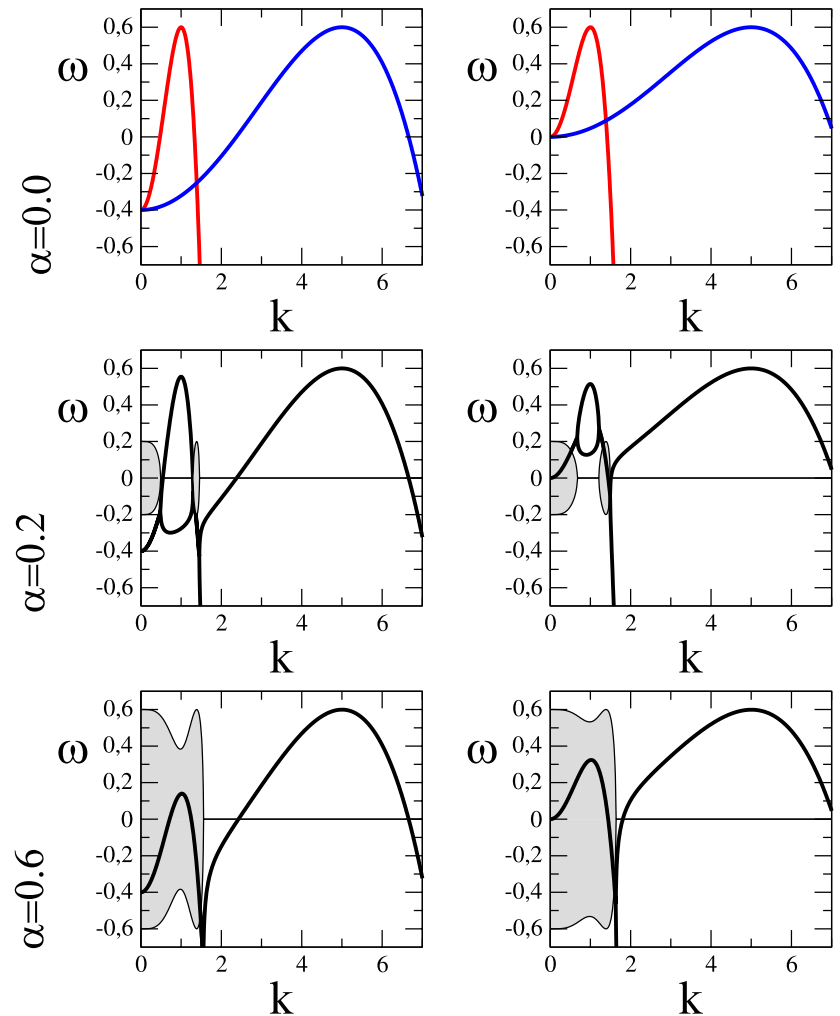

FIG. 2. Dispersion relation for two uncoupled (top) with $\alpha=0$ and coupled (middle) with $\alpha=0.2$ (bottom) with $\alpha=0.6 \mathrm{SH}$ (a) and $\mathrm{CH}$ (b) equations. Thick (thin) solid lines refer to real (imaginary) part of the eigenvalue $\omega$. Parameter values are $k_{1}=1$ and $k_{2}=5$ and $\varepsilon=0.6$.

leading eigenvalue becomes complex for low wavenumbers, see middle and bottom panel of Fig. 2(a).

\section{B. Coupled $\mathrm{CH}$ equations}

The standard $\mathrm{CH}$ equation is commonly used as a paradigmatic model of phase separation. ${ }^{28}$ In contrast to $\mathrm{SH}$ equation, it describes the evolution of a single conserved variable $u$

$$
\frac{\partial u}{\partial t}=\nabla^{2}\left(u^{3}-2 \varepsilon \frac{1}{k_{1}^{2}} u-\varepsilon \frac{1}{k_{1}^{4}} \nabla^{2} u\right) .
$$

Again, the spatially HSS $u_{0}=0$ is a stationary solution of the equation. By applying the perturbation $\delta u e^{\omega t-i k x}$ and by linearizing Eq. (5) around $u_{0}$, one obtains the dispersion relation as

$$
\omega(k)=2 \varepsilon \frac{k^{2}}{k_{1}^{2}}-\varepsilon \frac{k^{4}}{k_{1}^{4}} .
$$

For $\varepsilon>0$, the HSS become unstable for a finite interval of wavenumbers bounded from above from $k_{1}$, namely, for $0<k<\sqrt{2} k_{1}$. The characteristic spatial scale of the initial pattern is given by the most unstable wavenumber, namely, $\lambda_{1}=2 \pi / k_{1}$. For $\varepsilon<0$, the system is unstable for small spatial scales and higher order spatial derivatives are needed to stabilize the system. 
Analogously to the previous analysis, we linearly couple two $\mathrm{CH}$ equations in an asymmetric way, as follows:

$$
\begin{aligned}
& \frac{\partial u}{\partial t}=\nabla^{2}\left(u^{3}-2 \varepsilon \frac{1}{k_{1}^{2}} u-\varepsilon \frac{1}{k_{1}^{4}} \nabla^{2} u\right)-\alpha v, \\
& \frac{\partial v}{\partial t}=\nabla^{2}\left(v^{3}-2 \varepsilon \frac{1}{k_{2}^{2}} v-\varepsilon \frac{1}{k_{2}^{4}} \nabla^{2} v\right)+\alpha u .
\end{aligned}
$$

As for the coupled SH equations, we assume the same value of the control parameter $\varepsilon$ for both equations and the same coupling $\alpha$ with opposite signs. Once more we analyze the stability of the HSS $\left(u_{0}, v_{0}\right)=(0,0)$ by considering periodic perturbations to the vector $(u, v)$, namely, $\left(\delta u e^{\omega t-i k x}, \delta v e^{\omega t-i k x}\right)$. The linear stability analysis leads to the following dispersion relation:

$$
\begin{aligned}
\omega(k)= & \varepsilon k^{2} \frac{k_{1}^{2}+k_{2}^{2}}{k_{1}^{2} k_{2}^{2}}-\varepsilon k^{4} \frac{k_{1}^{4}+k_{2}^{4}}{2 k_{1}^{4} k_{2}^{4}} \\
& \pm \sqrt{\varepsilon^{2}\left[k^{2} \frac{k_{2}^{2}-k_{1}^{2}}{k_{1}^{2} k_{2}^{2}}-k^{4} \frac{k_{2}^{4}-k_{1}^{4}}{2 k_{1}^{4} k_{2}^{4}}\right]^{2}-\alpha^{2} .}
\end{aligned}
$$

As shown in Fig. 2(b), also in this case the coupled system exhibits two real maxima in the dispersion relation, resembling those of the uncoupled system. Furthermore, also in the present case we will denote the wavenumbers associated to these maxima as mode 1 at small $k$ and mode 2 at large $k$. The presence of the coupling between the two equations induces the emergence of imaginary components in the dispersion relation in the low wave vector part of the spectrum, as shown in Fig. 2(b).

\section{RESULTS}

In order to characterize the two previously introduced models, we first analyze their linear stability diagrams. In addition, we have performed extensive numerical simulations of the full nonlinear models.

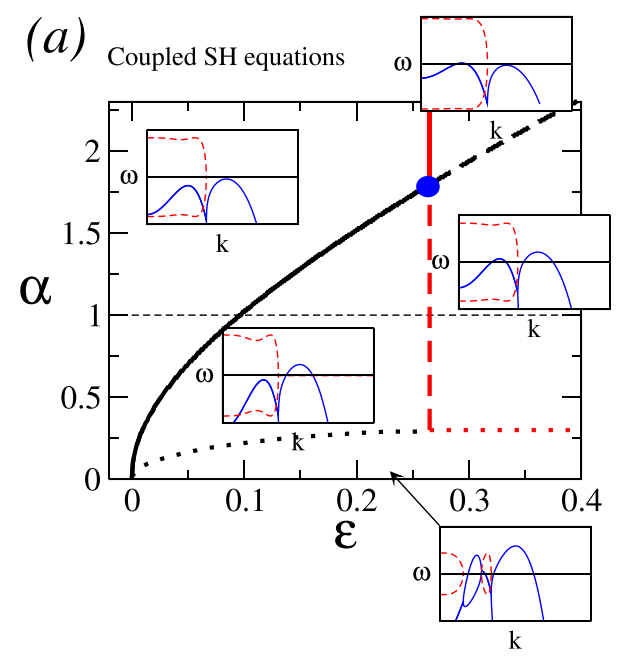

\section{A. Linear stability diagrams}

The linear stability analysis indicates that different types of behaviours are expected for different choices of the parameters $\varepsilon$ and $\alpha$. The linear stability diagrams for both systems are shown in Fig. 3. As a general remark, for small value of the coupling $\alpha$ both systems present spatial patterns. However, these are atypical spatial patterns, due to the coexistence of real modes 1 and 2 with unstable complex modes associated to non critical wavenumbers, see panels for $\alpha=0.2$ in Fig. 2.

The linear stability diagram for the coupled SH equations is shown in Fig. 3(a). For a large coupling constant $\alpha$ and small values of the control parameter $\varepsilon$, the HSS is stable. This state can lose stability in two different ways, depending $\alpha$ is larger or smaller than a critical value $\alpha_{c}$. For $\alpha>\alpha_{c}$, one observes a wave bifurcation involving mode 1 at

$$
\varepsilon_{c}=\frac{1}{2}-\frac{k_{1}^{2} k_{2}^{2}}{k_{1}^{4}+k_{2}^{4}},
$$

which corresponds to the solid red vertical line in Fig. 3(a). For $\alpha<\alpha_{c}$, the system undergoes a Turing instability for mode 2 (solid black line in Fig. 3(a)) and at $\varepsilon=\varepsilon_{c}$ an additional band of unstable oscillatory modes around mode 1 emerges (red dashed line in Fig. 3(a)). The two lines in the phase diagram, associated to these transitions, cross in a codimension-two point $\left(\varepsilon_{c}, \alpha_{c}\right)$ indicated by the blue dot in Fig. 3(a). For decreasing $\alpha$-values, at the dotted lines Fig. 3 (a), the eigenvalue associated to mode 1 passes from complex to real positive values, although other modes can still remain complex.

The linear stability diagram for the coupled $\mathrm{CH}$ equations is shown in Fig. 3(b). For positive $\varepsilon>0$, the system exhibits a wave bifurcation (red solid vertical line in Fig. 3(b)). Furthermore, at sufficiently large $\alpha$ values mode 1 is unstable and complex. By decreasing the $\alpha$ parameter, the system undergoes a secondary instability of mode 2 connected with real eigenvalues (black solid line in Fig. 3(b)).

\section{(b) Coupled CH equations}

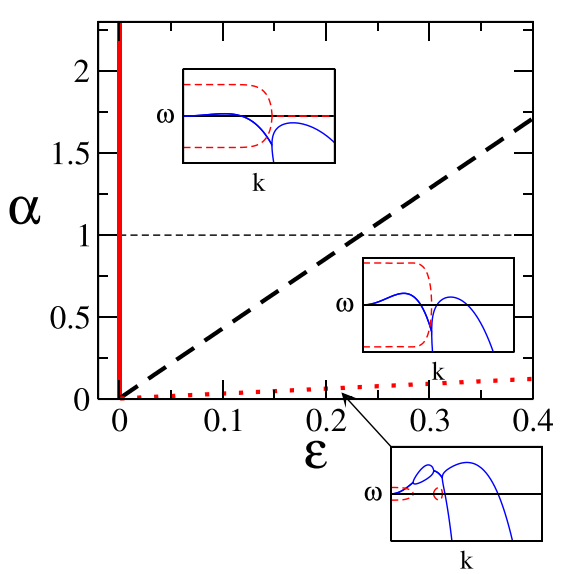

FIG. 3. Linear stability diagrams are given by Eqs. (4) and (8) for the parameters $\varepsilon$ and $\alpha$. Solid black (red) lines in (a) show Turing (wave) bifurcation, whereas the solid black lines in (b) correspond to emergence of an unstable band with finite wavenumber. The black (red) dashed lines indicate the emergence of a secondary Turing (wave) instability. Dotted lines mark the transition from real to complex maximum in the dispersion relation, see Fig. 2. Thick point indicates codimension-two point $\left(\varepsilon_{c} ; \alpha_{c}\right)$. Insets show characteristics dispersion relations in the corresponding region, where solid (dashed) lines correspond to real (imaginary) eigenvalues. Thin dashed horizontal lines are given for comparison with Fig. 4 . Remaining parameters are $k_{1}=1$ and $k_{2}=2$. 

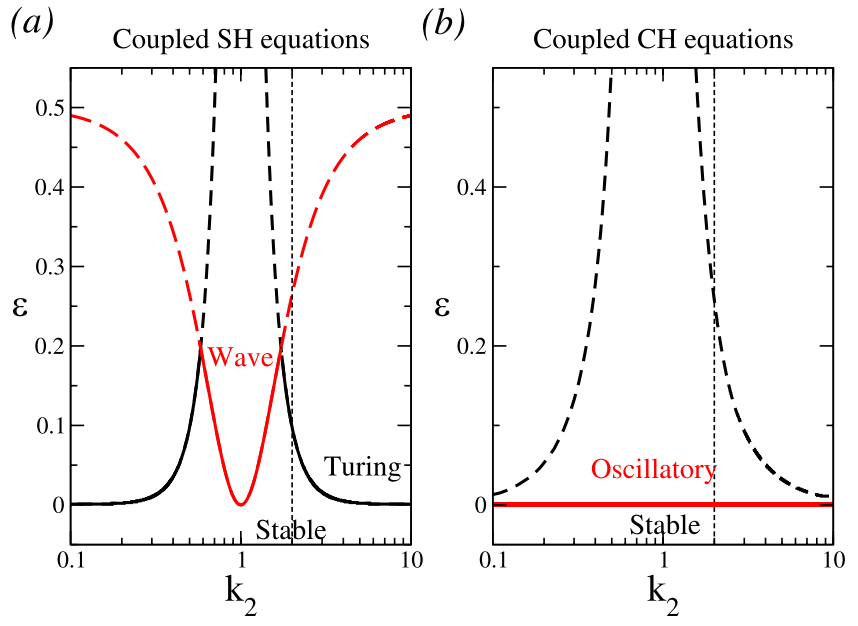

FIG. 4. Linear stability diagram are given by Eqs. (4) and (8) for the parameters $\varepsilon$ and $k_{2}$, keeping $\alpha=1$ and $k_{1}=1$, for two coupled SH equations (a), and two coupled $\mathrm{CH}$ equations (b). The lines have the same meaning as in Fig. 3. Thin dashed vertical lines refer to the parameter $k_{2}$ employed in Fig. 3.

Below this black line, these two types of unstable modes coexist. At sufficiently small $\alpha$ values, mode 1 becomes purely real (dotted red line in Fig. 3(b)), although other modes are still complex.

In Fig. 4, we systematically change the ratio between the two parameters $k_{1}$ and $k_{2}$ for the two models. For the special case $k_{1}=k_{2}$, the traveling solution is always present independently of the value of the other parameters (since the imaginary components of $\omega(k)$ in Eqs. (4) and (8) is in this case constant and independent of $k$ ). The linear stability diagrams are symmetric around the point $k_{1}=k_{2}$.

For the coupled SH equations, the appearance of traveling solutions does not depend on the coupling strength but on the control parameter $\varepsilon$ (red lines in Fig. 4(a)). The critical value of the $\varepsilon$ parameter, controlling the emergence of waves, depends on the relation between the two scales $k_{1}$ and $k_{2}$, and it can be analytically calculated from Eq. (9). For $k_{2} \gg k_{1}$, the secondary instability occurs at $\varepsilon_{c}=0.5$, see red dashed line in Fig. 4(a). In between $0<\varepsilon_{c}<0.5$, the dynamics depends on the coupling and $k_{2}$.

The linear stability diagram for the coupled $\mathrm{CH}$ equations is a simplified version of the previous case, as one can appreciate by comparing panels (a) and (b) in Fig. 4. The wave instability is always present, at least for not too large values of $\varepsilon$, however, large $k_{2}$ enhances the appearance of the spatial instability and promotes the competition between both instabilities.

\section{B. Numerical simulations}

We have employed a time splitting pseudo spectral method, similar to the one described in Ref. 29, to numerically integrate Eqs. (3) and (7). The simulations have been performed by considering mainly one dimensional systems with size $L=40 \pi$ or $L=80 \pi$ with periodic boundary conditions. For the numerical integration 512 or 1024 Fourier modes have been alternatively used and integration time steps in the range $\Delta t=0.001-0.01$. The runs are usually initialized by

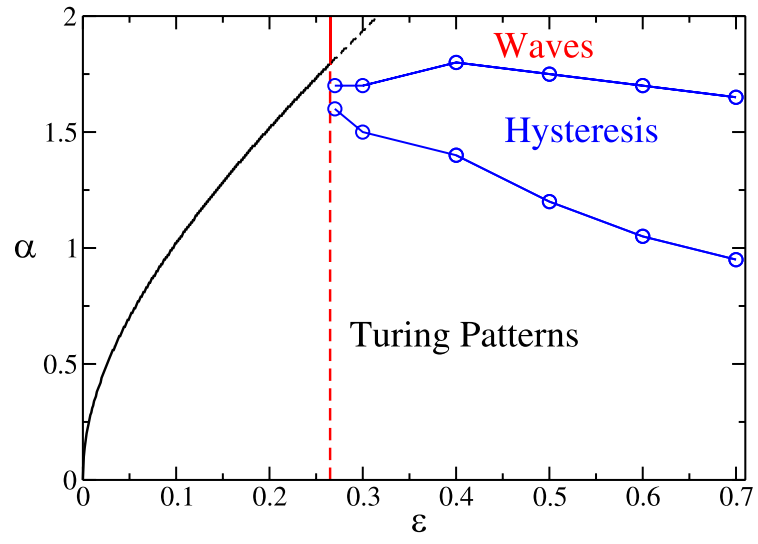

FIG. 5. Phase diagram $(\varepsilon ; \alpha)$ for the coupled SH Eqs. (3) estimated numerically. For the details on the numerical simulation see the text. The solid and dashed black (red) lines refer to the results of the linear stability analysis and have the same meaning as in Fig. 3. The blue line with symbols denote the limits if the hysteretic region. Parameter values are $k_{1}=1$ and $k_{2}=2$, the system has been integrated for each couple of $(\varepsilon, \alpha)$ parameters for a time 20000 with a time step $\Delta t=0.001$ by considering a system size $L=40 \pi$ and by employing 512 Fourier modes.

setting $u$ and $v$ to random values uniformly distributed in the interval $[-1 ; 1]$.

The numerical simulations reproduce the results predicted by the linear stability analysis for small values of $\alpha$, while for larger coupling $\alpha$ nonlinear effects come into play leading to a richer scenario not predicted by linear analysis. Here, we focus on the competition between waves and Turing patterns revealed by the coupled SH equations and on the arrest of coarsening occurring in the coupled $\mathrm{CH}$ equations.

\section{Coupled SH equations}

The numerically obtained phase diagram for the coupled SH equations is shown in Fig. 5, which has been obtained by keeping constant $\varepsilon(\alpha)$ (for a certain set of values) and by varying the other parameter, namely $\alpha(\varepsilon)$. The parameter $\alpha$ $(\varepsilon)$ is first increased and successively decreased of a constant amount $\Delta \alpha=0.05(\Delta \varepsilon=0.05)$. Each simulation had a duration of 2000-20000 time units and the next simulation in the sequence is initialized by employing the final state of the previous one. This allows to reveal a hysteretic transition in the $(\varepsilon, \alpha)$-plane for $\varepsilon>\varepsilon_{c}$ : the corresponding hysteretic region is enclosed by the blue curves in Fig. 5. Therefore, within this region traveling waves or Turing patterns can be observed, depending on the initial conditions.

Some examples of Turing patterns and waves obtained in the simulations are reported in Fig. 6. When both systems are weakly coupled, i.e., small $\alpha$ in Fig. 5, two distinct Turing patterns characterized by different spatial scales can be observed in variables $u$ and $v$ (Fig. 6(a)). For larger $\alpha$, the coupling eliminates the instability with smaller characteristic scale and generates a wave instability associated to the larger spatial scale in both variables, see Fig. 6(b). Deep inside the hysteretic region one can observe the coexistence of Turing patterns, embedded in traveling waves (as shown in Fig. 6 (c)). For very large $\varepsilon \simeq 0.9$ spatio-temporal chaotic irregular dynamics with defects is observable (see Fig. 6(d)) and 
(a)

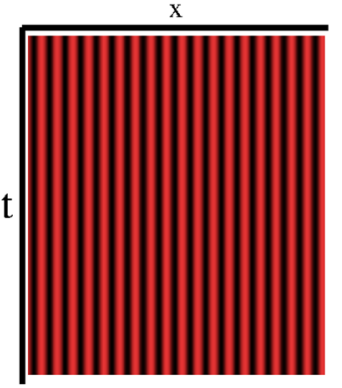

(b)

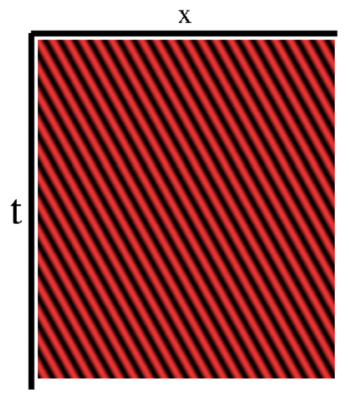

(c)

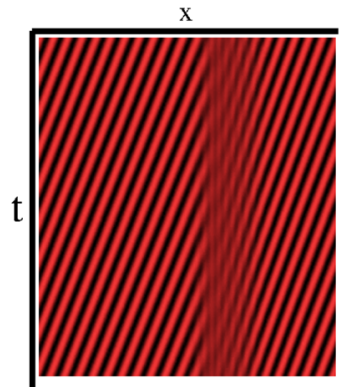

(d)

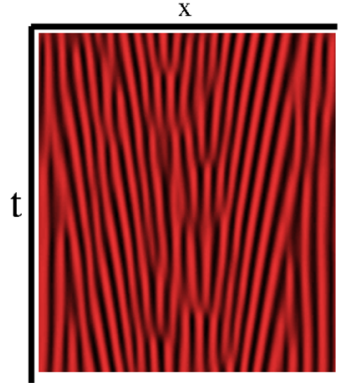

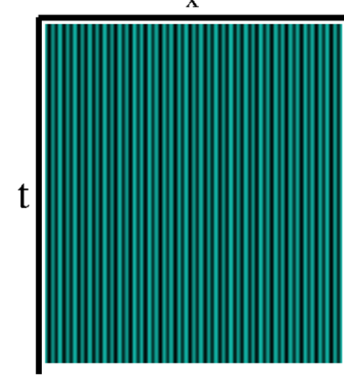
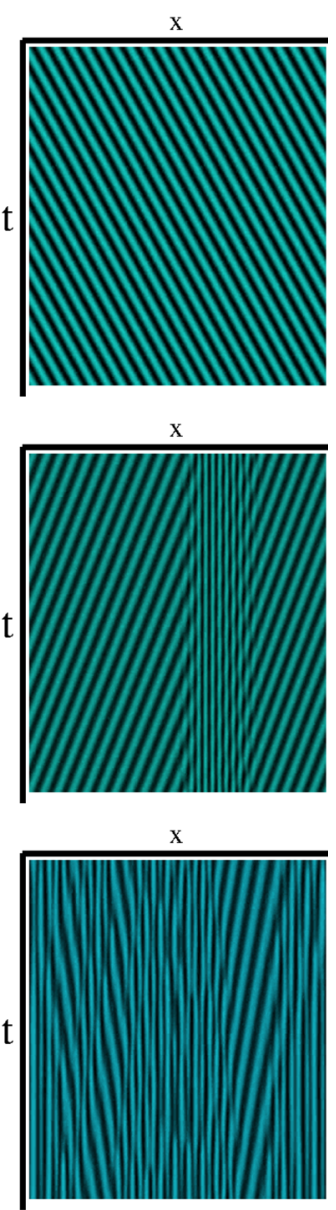

FIG. 6. Spatio-temporal plots of the dynamics of $u$ (left) and v (right) variables obtained after numerical integration of the coupled SH Eqs. (3) for the parameters $\varepsilon=0.3$ and $\alpha=0.3$ (a), $\varepsilon=0.4$ and $\alpha=1.6$ (b), $\varepsilon=0.7$ and $\alpha=1.2$ (c), and $\varepsilon=0.9$ and $\alpha=0.6$ (d). Rest of parameter values are $k_{1}=1$ and $k_{2}=2$. The integration time is 50 , after discarding a transient of 2000 with $\Delta t=0.001$, and the system size is set to $L=40 \pi$ with a spatial discretization $\Delta x=L / 512 \simeq 0.245$. Time direction is going downwards in all panels.

we have verified that this is not a transient regime by performing long simulations up to time $t \simeq 50000$.

\section{Coupled $\mathrm{CH}$ equations}

Examples of patterns found in simulations of the coupled $\mathrm{CH}$ equations, for increasing coupling parameter $\alpha$ are shown in Fig. 7. For small $\alpha$, one observes initial coarsening in the $u$ and $v$ variables similar to what is found in the uncoupled single $\mathrm{CH}$ equations in Fig. 7(a). As expected from the linear stability analysis above, the initial domain patterns of $u$ and $v$ have different characteristic wavelength. However, the coarsening process stops after a finite time and

(a)
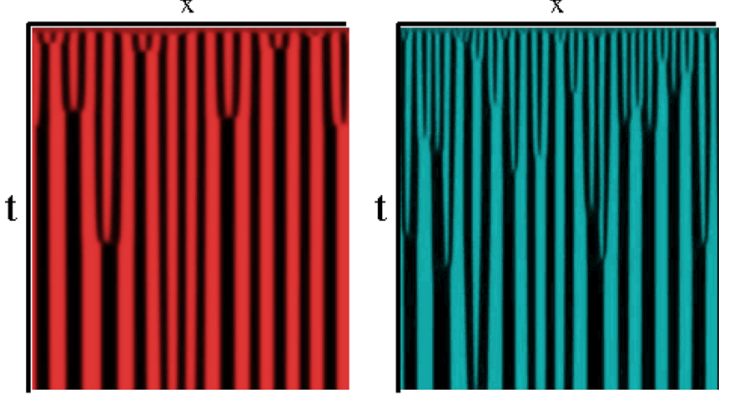

(b)
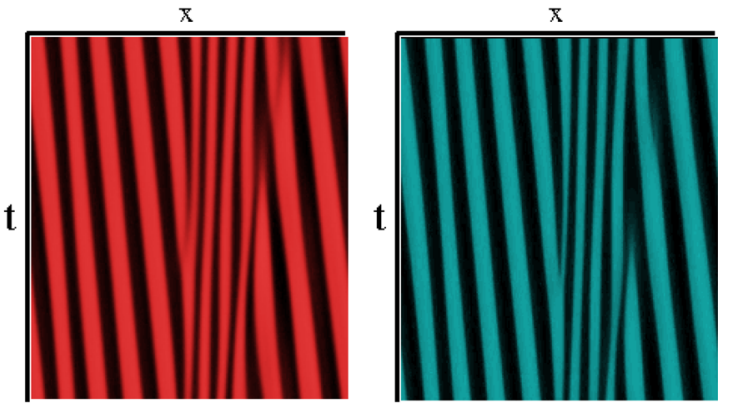

(c)
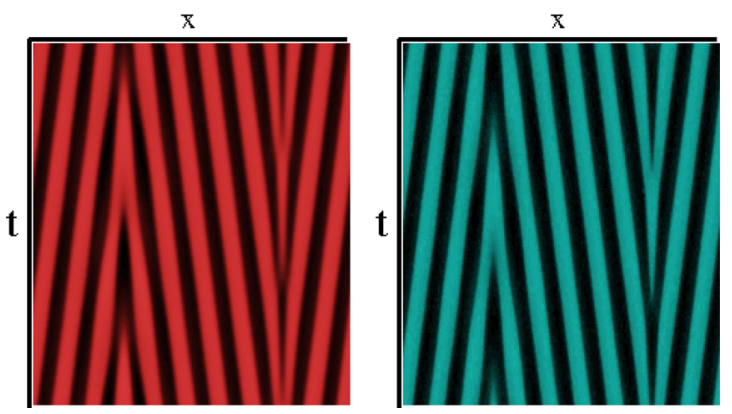

FIG. 7. Spatio-temporal plots of the dynamics of the $u$ (left) and $v$ (right) variables obtained after numerical integration of the coupled $\mathrm{CH}$ Eqs. (7) for the parameters $\varepsilon=0.5$ and $\alpha=0.002$ (a), $\alpha=0.15$ (b), and $\alpha=0.2$ (c). The integration time is 1500 without any transient for (a) and 50 after a transient of 2000 for (b) and (c) with $\Delta t=0.001$, and system size is $L=40 \pi$ and spatial discretization $\Delta x=L / 512=\simeq 0.245$. Time direction is going downwards in all panels.

both variables- $u$ and $v$-exhibit a domain pattern of the same wavelength. For larger values of $\alpha$, traveling waves are observed in line with the occurrence of oscillatory unstable modes in the linear stability analysis, see Figs. 7(b) and 7(c).

Figure 8 shows typical spatial patterns for $u$ and $v$ emerging at long integration times for the different cases. In particular, in Fig. 8(a) are reported the solutions for the uncoupled equations, Fig. 8(b) shows patterns with equal wavelength for $u$ and $v$ in the case of arrested coarsening (occurring at small $\alpha$ ), and Fig. 8(c) reports left-traveling domains appearing at large $\alpha$.

As already shown in Fig. 7(a), on short time scales one has the typical dynamics of the single Cahn-Hilliard equation, i.e., a coarsening process. However, the subsystem with larger wavelength coarsens faster than the other variable. This process continues until the two variables $u$ and $v$ lock into periodic patterns of the same characteristic length $L_{c}$ (see Fig. 9(a)). Once the two variables have taken on the same wavelength, the coarsening process stops and $L_{c}$ remains constant. In this case, the profile of the two variables is perfectly periodic with maxima and minima of both 


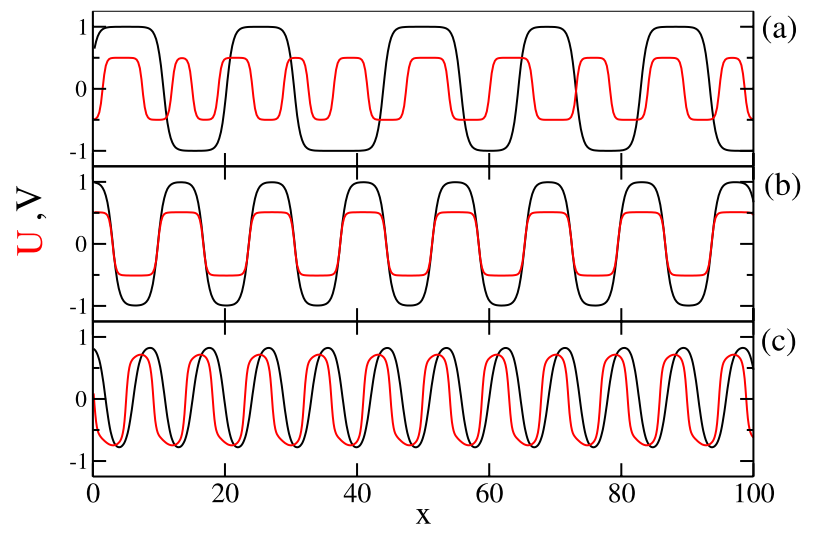

FIG. 8. Characteristic patterns for the $u, v$ variables in one spatial dimension obtained by numerical integration of Eqs. (7) for parameters $\varepsilon=0.5$ and $\alpha=0$ (a), $\alpha=0.002$ (b), $\alpha=0.2$ (c). The systems size is $L=80 \pi$ and the integration is performed by employing a time splitting pseudo-spectral code with 1024 Fourier modes and an integration time step $\Delta t=0.01$. The configurations in (a) refer to an integration time $T=41942$, while those in (b) and (c) to a time $t=671088$.

variables occurring in phase as shown in Fig. 8(b). For comparison, the evolution of the two uncoupled systems is reported in Fig. 9(b), where coarsening is not arrested during the simulation time window and the scaling of $L_{c}$ grows logarithmically in time as expected for a one-dimensional $\mathrm{CH}$ equation in the absence of noise. ${ }^{30}$ Furthermore, the spatial configurations for the variables $u$ and $v$ do not lock even at very long times, see Fig. 9(b).

We have also investigated the scaling of the time of arrest $T^{A}$ of coarsening with the coupling parameter $\alpha$. As shown in Fig. 10(a), a power-law scaling of the type $T^{A} \simeq \alpha^{-\eta}$, with $\eta$ $\simeq 0.8-0.9$, is observable. Furthermore, the coarsening process is arrested at increasing characteristic lengths $L_{c}^{A}$ diverging as $\ln (1 / \alpha)$ for decreasing $\alpha$-values, see Fig. 10(b). These two scaling laws are essentially consistent with the logarithmic coarsening in time reported for the deterministic one dimensional $\mathrm{CH}$ equation, thus suggesting that the asymptotic value for the exponent $\eta$ should be one.

An arrest of coarsening has been previously reported for scalar fields in one spatial dimension for the Oono-Shiwa model. ${ }^{25}$ This is a modified version of the single $\mathrm{CH}$ equation,

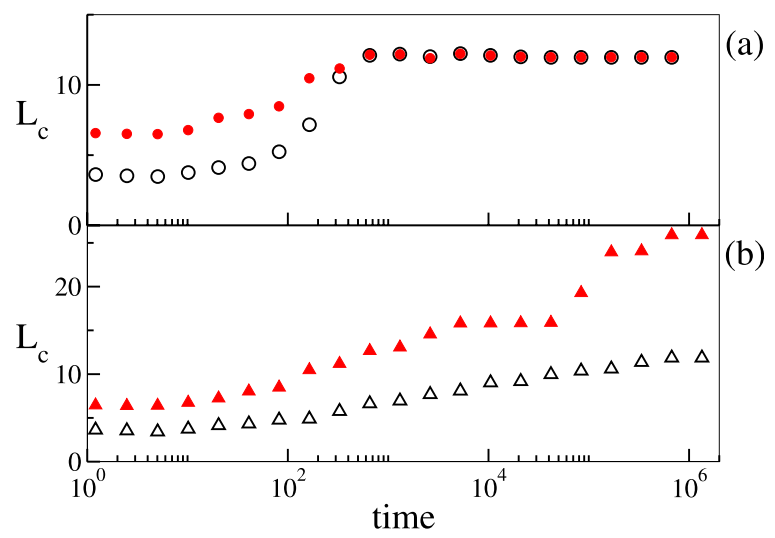

FIG. 9. Characteristic size $L_{c}$ of the domains obtained by numerical integration of Eqs. (7) for the parameters $\varepsilon=0.5$ and $\alpha=0.002$ (a), $\alpha=0.0$ (b). The (red) filled symbols refer to variable $u$, and the (black) empty to $v$. The system size and integration details are as in Fig. 8.

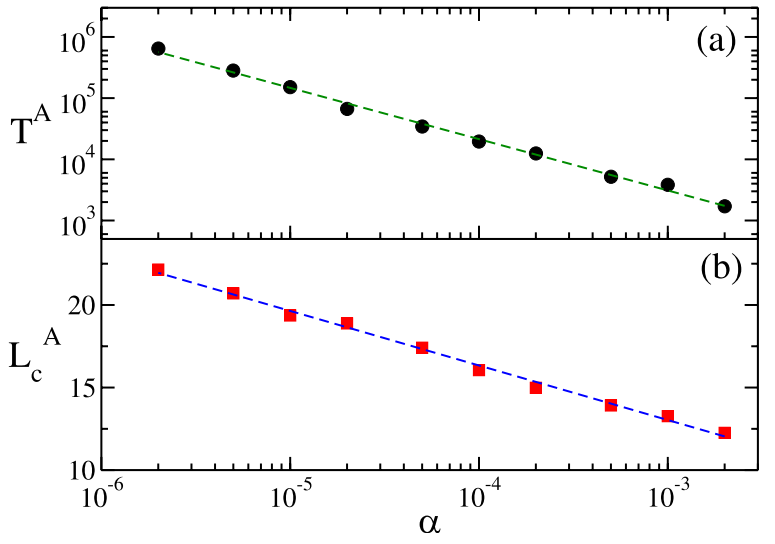

FIG. 10. Average arrest time $T^{A}$ (a) and average arrest length $L_{c}^{A}$ (b) as a function of the coupling $\alpha$. The averages have been performed over 20-60 different initial conditions. The standard deviation measured in the case of $T^{A}$ is of the order of the averages and in the case of $L_{c}^{A}$ of the order of $10 \%$ of the averages. The dashed lines indicate power-law (logarithmic) fitting to the data for $T^{A}\left(L_{c}^{A}\right)$, namely, $T_{A} \simeq 9.5 \alpha^{-0.84}\left(L_{A}^{c} \simeq 3.123-1.435 \ln (\alpha)\right)$. The system size and integration details are as in Fig. 8 .

with an additional linear coupling to the order parameter, which has been developed to mimic phase separation in block copolymer melts. ${ }^{25,31,32}$ The analysis of this model revealed that the system arrests and gives rise to periodic regular stable structures, similar to what we observe here for the coupled $\mathrm{CH}$ equations. However, while the arrest of coarsening in the Oono-Shiwa model is due to the stabilization of longwavelength modes by the additional linear term, this effect is not present for the coupled $\mathrm{CH}$ equations studied here.

Instead, the arrest of coarsening stems from the interaction of the $u$ and $v$ fields. The presence of the initially longer wavelength mode in $u$ accelerates the coarsening of initially the shorter wavelength modes in $v$ much more than vice versa. Hence, one can say that each pattern acts as a template for the other one and as a result coarsening stops. This is reminiscent of domain pinning seen in dewetting processes on heterogeneous substrates, ${ }^{33,34}$ or of spatial forcing as seen in single $\mathrm{CH}$ equations mimicking optical grating experiments, ${ }^{35}$ CDIMA photosensitive reactions, ${ }^{36}$ and single $\mathrm{SH}$ equations. ${ }^{37}$

\section{CONCLUSIONS}

We examined coupled $\mathrm{SH}$ and the $\mathrm{CH}$ equations as simple models for pattern formation in systems with competing instabilities of different characteristic wavelength. While a single $\mathrm{SH}$ o $\mathrm{CH}$ equation exhibits only instabilities connected with real eigenvalues and modes and, therefore, can only produce stationary periodic spatial patterns $(\mathrm{SH})$ or a slow domain coarsening $(\mathrm{CH})$, the asymmetric coupling of two equations typically leads to the occurrence of oscillatory unstable modes with complex eigenvalues and, consequently, to the emergence of traveling waves and domains.

Using linear stability analysis, we determine the conditions for the occurrence of stationary and oscillatory instabilities and their dependence on the parameters in the coupled SH and $\mathrm{CH}$ equations. In particular, the choice of opposite signs in the coupling produces the appearance of complex eigenvalues in the dispersion relations, see Eqs. (4) and (8). At the same time, this choice does not allow to rewrite the 
coupled system as a variational derivative of an associated energy functional.

The linear stability analysis is complemented by numerical simulations of the full nonlinear models in a wide parameter range. These studies reveal a region of coexistence of traveling waves and Turing patterns in the coupled $\mathrm{SH}$ equations. Furthermore, a rich variety of patterns has been observed ranging from traveling waves with entrapped Turing patterns to one-dimensional defect turbulence.

On the other hand, for coupled $\mathrm{CH}$ equations the logarithmic coarsening, typical of the one-dimensional single $\mathrm{CH}$ equations, is arrested even in presence of a very small coupling. The spatial patterns of the two variables coarsen at different velocities. The pattern with smaller characteristic wavelength coarsens faster than the one with longer wavelength until the profiles of the two patterns attain the same spatial periodicity and are locked in space. As a result, coarsening stops and the wavenumber stays constant for arbitrary long times: the final domain pattern is stable and stationary in time. The corresponding final state for the two variables has the same wavelength, but different amplitude profiles. Furthermore, the asymptotic characteristic length diverges logarithmically for vanishingly small coupling.

Arrest of coarsening has been previously reported for scalar fields in one spatial dimension for a modified version of the single specie $\mathrm{CH}$ equation developed to mimic phase separation in block copolymer melts. ${ }^{25,31,32}$ In this case, the final state reveals a periodic stable structure. On the contrary, the arrest of coarsening reported in Ref. 38, was associated to an unstable asymptotic pattern with a diverging amplitude. In two dimensions arrest of coarsening has been shown in Ref. 39 for spinodal decomposition of mixtures in presence of an externally controlled chemical reaction, in Ref. 40 for a $\mathrm{CH}$, where the order parameter is subjected to an external stirring, both for active and passive mixtures, and in Ref. 41 for a modified $\mathrm{CH}$, where the order parameter is coupled linearly to the Langevin equations describing the dynamics of Janus particles.

The results obtained here are analogous to what is found in more complex models describing lipid and protein dynamics at membranes of biological cells ${ }^{17,42}$ where waves have been observed following similar mechanisms. Since the study of the single $\mathrm{CH}$ equation linearly coupled to the order parameter has been motivated by phase separation in block copolymers melt, ${ }^{25}$ the analysis of two linearly coupled $\mathrm{CH}$ equations can eventually find application in the study of selfdirected self-assembly observed in mixtures of copolymers and nanoparticles. ${ }^{43}$ Possible extensions of this study may consider the conserved $\mathrm{SH}$ equation ${ }^{44}$ where higher spatial derivatives are included.

In summary, a simple linear asymmetric coupling between two nonlinear equations may produce substantial changes in the dynamics, producing waves, chaotic dynamics, hysteresis, or arrest of coarsening.

\section{ACKNOWLEDGMENTS}

We thank S. Lepri and P. Politi for a useful exchange of ideas and M. Cencini for a careful reading of this manuscript prior to the submission. We acknowledge financial support from the German Science Foundation DFG within the framework of SFB 910 "Control of self-organizing nonlinear systems." A.T. has been partially supported by the Italian MIUR project CRISIS LAB PNR 2011-2013.

${ }^{1}$ M. C. Cross and P. C. Hohenberg, Rev. Mod. Phys. 65, 851 (1993).

${ }^{2}$ Chemical Waves and Patterns, edited by R. Kapral and K. Showalter (Kluwer, Dordrecht, 1994).

${ }^{3}$ J. D. Murray, Mathematical Biology (Springer, 2002).

${ }^{4}$ J. Keener and J. Sneyd, Mathematical Physiology (Springer, New York, 2009).

${ }^{5}$ A. M. Turing, Philos. Trans. R. Soc. London, Ser. B 237, 37 (1952).

${ }^{6}$ S. Hata, H. Nakao, and A. S. Mikhailov, Prog. Theor. Exp. Phys. 2014, $013 \mathrm{~A} 01$.

${ }^{7}$ A. De Wit, D. Lima, G. Dewel, and P. Borckmans, Phys. Rev. E 54, 261 (1996).

${ }^{8}$ M. Meixner, A. De Wit, S. Bose, and E. Schöll, Phys. Rev. E 55, 6690 (1997).

${ }^{9}$ W. Just, M. Bose, S. Bose, H. Engel, and E. Schöll, Phys. Rev. E 64, 026219 (2001).

${ }^{10}$ L. Yang, A. M. Zhabotinsky, and I. R. Epstein, Phys. Rev. Lett. 92, 198303 (2004).

${ }^{11}$ L. F. Yang, M. Dolnik, A. M. Zhabotinsky, and I. R. Epstein, Phys. Rev. Lett. 88, 208303 (2002).

${ }^{12}$ S. Kinast, Y. R. Zelnik, G. Bel, and E. Meron, Phys. Rev. Lett. 112, 078701 (2014).

${ }^{13}$ L. Yang, M. Dolnik, A. M. Zhabotinsky, and I. R. Epstein, J. Chem. Phys. 117, 7259 (2002).

${ }^{14}$ S. Alonso, K. John, and M. Bär, J. Chem. Phys. 134, 094117 (2011).

${ }^{15}$ K. John, S. Alonso, and M. Bär, Phys. Rev. E 90(5), 052913 (2014).

${ }^{16}$ K. John and M. Bär, Phys. Rev. Lett. 95, 198101 (2005).

${ }^{17}$ K. John and M. Bär, Phys. Biol. 2, 123 (2005).

${ }^{18}$ S. Alonso and M. Bär, Phys. Biol. 7, 046012 (2010).

${ }^{19}$ J. Swift and P. C. Hohenberg, Phys. Rev. A 15, 319-328 (1977).

${ }^{20}$ J. Swift and P. C. Hohenberg, Scholarpedia 3, 6395 (2008).

${ }^{21}$ A. Hutt and F. M. Atay, Phys. D 203, 30 (2005).

${ }^{22}$ J. Lega, J. Moloney, and A. Newell, Phys. Rev. Lett. 73, 2978 (1994).

${ }^{23}$ J. W. Cahn and J. E. Hilliard, J. Chem. Phys. 28, 258 (1958).

${ }^{24}$ S. L. Veatch and S. L. Keller, Biophys. J. 85, 3074-3083 (2003).

${ }^{25}$ Y. Oono and Y. Shiwa, Mod. Phys. Lett. B 1, 49 (1987).

${ }^{26}$ L. Yang, M. Dolnik, A. M. Zhabotinsky, and I. R. Epstein, Chaos 16, 037114 (2006).

${ }^{27}$ J. Li, H. Wang, and Q. Ouyang, Chaos 24, 023115 (2014).

${ }^{28}$ R. C. Desai and R. Kapral, Dynamics of Self-organized and Selfassembled Structures (Cambridge University Press, 2009).

${ }^{29}$ A. Torcini and P. Politi, Eur. Phys. J. B 25, 519-529 (2002).

${ }^{30}$ P. Politi and A. Torcini, J. Phys. A: Math. Gen. 33, L77 (2000).

${ }^{31}$ F. Liu and N. Goldenfeld, Phys. Rev. A 39, 4805 (1989).

${ }^{32}$ S. Villain-Guillot, Discontinuity and Complexity in Nonlinear Physical Systems, Nonlinear Systems and Complexity, edited by J. A. T. Machado et al. (Springer International, 2014), Vol. 6, p. 153.

${ }^{33}$ L. Brusch, H. Kühne, U. Thiele, and M. Bär, Phys. Rev. E 66, 011602 (2002).

${ }^{34}$ U. Thiele, L. Brusch, M. Bestehorn, and M. Bär, Eur. Phys. J. E 11, 255-271 (2003).

${ }^{35}$ A. P. Krekhov and L. Kramer, Phys. Rev. E 70, 061801 (2004).

${ }^{36}$ S. Rüdiger, D. G. Mguez, A. P. Muñuzuri, F. Sagués, and J. Casademunt, Phys. Rev. Lett. 90, 128301 (2003).

${ }^{37}$ Y. Mau, A. Hagberg, and E. Meron, Phys. Rev. Lett. 109, 034102 (2012).

${ }^{38}$ P. Politi and C. Misbah, Phys. Rev. Lett. 92, 090601 (2004).

${ }^{39}$ S. C. Glotzer, E. A. Di Marzio, and M. Muthukumar, Phys. Rev. Lett. 74, 2034 (1995).

${ }^{40}$ S. Berti, G. Boffetta, M. Cencini, and A. Vulpiani, Phys. Rev. Lett. 95, 224501 (2005).

${ }^{41}$ A. Krekhov, V. Weith, and W. Zimmermann, Phys. Rev. E 88, 040302 (2013).

${ }^{42}$ G. Meacci and K. Kruse, Phys. Biol. 2, 89 (2005).

${ }^{43}$ Y. Lin et al., Nature 434, 55 (2005).

${ }^{44}$ U. Thiele, A. J. Archer, and M. J. Robbins, Phys. Rev. E 87, 042915 (2013). 